\author{
Brief article
}

\title{
Effects of physical connectivity on the representational unity of multi-part configurations
}

\author{
Robert van Lier ${ }^{\mathrm{a}, \mathrm{b}, *}$, Johan Wagemans ${ }^{\mathrm{b}}$ \\ ${ }^{a}$ Nijmegen Institute for Cognition and Information, University of Nijmegen, \\ P.O. Box 9104, NL-6500 HE Nijmegen, The Netherlands \\ ${ }^{\mathrm{b}}$ University of Leuven, Leuven, Belgium
}

\begin{abstract}
While physical connection strongly supports perceptual integration of visual elements or parts into single objects, concavities along an object's contour strongly induce segmentation of objects into different parts. By means of a matching task, we examine whether the segmentation of connected parts and the grouping of disconnected parts imply differences in representational unity. All match trials consisted of either two identical configurations or two $180^{\circ}$ rotated versions of an otherwise identical configuration, whereas mismatch trials had one misoriented part. Results show that the $180^{\circ}$ matches with the physically disconnected parts were relatively slower than similar matches with physically connected parts, although the connections could be minimal (deep concavities, corner to edge connection). This suggests that the mental operation required for the $180^{\circ}$ match has more difficulty with the disconnected parts, indicating a measurably lower degree of representational unity. (C) 1998 Elsevier Science B.V. All rights reserved
\end{abstract}

Keywords: Perceptual organization; Shape matching; Structural description; Perceptual objects; Wholes and parts; Proximity; Connectedness; Concavity/convexity

When interpreting the visual environment, the perceptual system almost instantaneously groups and segments patches of light into an understandable world. Perhaps the most compelling clue that object parts belong together is physical connection. Such connectivity can be considered as an extreme case of proximity (e.g. Kubovy et al., 1998; Kubovy and Wagemans, 1995; Wertheimer, 1923), one of the classic Gestalt grouping factors. According to Palmer and Rock (1994), physical connection is even more powerful than close proximity, and actually differs from it,

* Corresponding author. E-mail: r.vanlier@nici.kun.nl 
as it may induce new perceptual units. In addition, Saiki and Hummel (1998) recently reported that connection of object parts facilitates the perception of specific spatial relations (such as left, right, above, below) between the parts; a finding which is consistent with the alleged special status of physical connection.

In contrast to grouping, objects are often perceptually segmented into separate parts despite their physical connection. In such segmentations, contour discontinuities are known to play an important role, especially at regions of concavity (e.g. Baylis and Driver, 1995a,b; Hoffman and Richards, 1984; Hoffman and Singh, 1997; Lamote and Wagemans, 1998; Marr and Nishihara, 1978; Siddiqi et al., 1996). According to Baylis and Driver (1995a,1995b) segmentations at concavities occur obligatorily and preattentively. This well-established phenomenon has a great impact on contemporary theorizing of shape perception and object recognition. In the Recognition-by-Components (RBC) model of Biederman (1987), for example, volumetric object parts (i.e. geons) segmented at concavities are taken as different entities that serve as building blocks to recognize objects. These geons and their spatial relations also play an important role in the neural network of shape recognition (JIM) of Hummel and Biederman (1992). However, JIM thus far does not discriminate between connected and disconnected geons (p. 514).

In this paper, we examine whether, for a given configuration, the grouping of disconnected parts and the segmentation of connected parts induce the same or different degrees of representational unity of the whole configuration. It was shown by Baylis and Driver (1993) that between-objects judgements are more difficult than within-objects judgements. Our question here is whether segmentation at concavities and physical disconnection induce the same or a different degree of multi-objectness. To investigate this issue, we have introduced a matching task in which participants were asked to compare two multi-part configurations in which the parts are either physically connected or physically disconnected. In a match trial, the configurations could be the same or they could be $180^{\circ}$ rotated versions of each other. Mismatch trials always differed in the orientation of one of the configural parts. If physical disconnection decreases the representational unity of the parts more than already induced by the concavities, then we would expect that the latter comparisons are more difficult and more time consuming than holistic comparisons (e.g. Bethell-Fox and Shepard, 1988).

\section{Methods}

\subsection{Participants}

Nine undergraduate students participated in the experiment to receive course credit. They were all naive with respect to the goal and the design of the experiment.

\subsection{Stimuli}

All stimuli were made of the same three parts; a large isosceles triangle (T), a 
trapezoid (Tz), and a small isosceles triangle (t). In Fig. 1A, the three parts are shown proportionally to their actual sizes on the monitor screen. The lengths of the horizontal sides were all different from one another. There were three classes of stimuli: connected, disconnected, and control. In Fig. 1B, the $0^{\circ}$ and $180^{\circ}$ versions of one specific arrangement are shown for each of these classes. Whereas the parts in the connected class just 'touch' each other, the parts in the disconnected class are separated from each other by a small distance of less than $5 \%$ of the height of the connected configuration. In the control class, the three parts are also connected with each other, but now the shape of the individual parts are accentuated by a black outline making them clearly distinguishable.

The set of stimuli was constructed by having all possible positional permutations of the three parts in a vertical order, having a vertical axis of symmetry. An order of parts could be one of the following: T-Tz-t, T-t-Tz, Tz-T-t, t-Tz-T, Tz-t-T and t-TTz. Note that for each order of parts, there is an inverse order (compare the first three
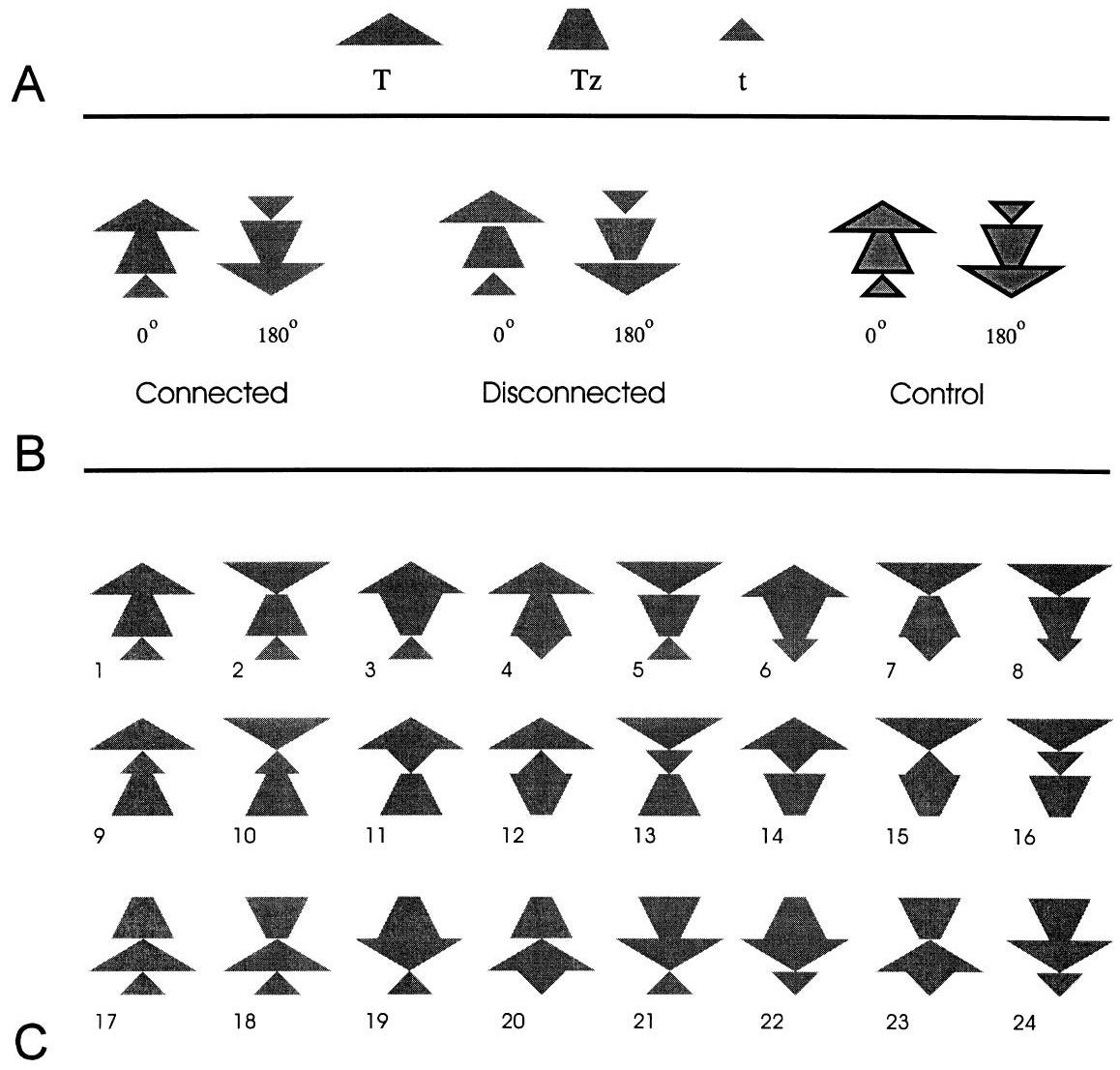

Fig. 1. (A) The three parts of which all configurations were made. (B) From each of three classes of configurations (connected, disconnected, and control) one specific configuration is shown in two different orientations $\left(0^{\circ}\right.$ and $\left.180^{\circ}\right)$. (C) All configurations within the connection class (shown in 1 of two orientations). 
orders versus the last three orders). So, in fact, there are three different arrangements of parts. Furthermore, each single part was positioned in two different ways; pointing upwards or pointing downwards. For each of the above orders of parts, this manipulation yields eight different compositions $(2 \times 2 \times 2)$. So, for example, for the order T-Tz-t this leads to the following compositions (in the notation the prime indicates an inversion of the specific part): T-Tz-t, $T^{\prime}-T z-t, T-T z^{\prime}-t, T-T z-t^{\prime}, T^{\prime}-T z^{\prime}-t$, $\mathrm{T}-\mathrm{T} z^{\prime}-\mathrm{t}^{\prime}, \mathrm{T}^{\prime}-\mathrm{Tz}-\mathrm{t}^{\prime}$, and $\mathrm{T}^{\prime}-\mathrm{T} z^{\prime}-\mathrm{t}^{\prime}$. In total, this stimulus construction procedure results in 48 different compositions within each of three classes. Of these 48 compositions, there are essentially 24 different configurations. That is, each configuration appears in two different orientations $\left(0^{\circ}\right.$ and $180^{\circ}$, i.e. upright or inverted). In Fig. 1C, all 24 configurations are shown the way they appear in the connected class (in one of two orientations).

This construction of an exhaustive set of configurations consisting of the same three parts (based on permutations and inversions) implies that the actual connections vary between configurations. Because all horizontal edges were of different length, all connections reveal concavities between parts. Notice that in half of the configurations $(1,2,5,8,9,10,13,16,17,18,21$ and 24), all concavities are deep (i.e. external angle $<90^{\circ}$ ), whereas in the other half, one pair of concavities is deep whereas the other is not (i.e. $90^{\circ}<$ external angle $<180^{\circ}$ ). Notice further that for the connected class several connections between the parts can be distinguished; edge to edge (EE), corner-to-edge (CE), and corner to corner (CC). Within the constructed set of configurations, the following combinations occur (see Fig. 1C): $\mathrm{EE} / \mathrm{EE}(4,6), \mathrm{EE} / \mathrm{CE}(1,3,7,8,9,11,12,14,20,22,23,24) \mathrm{EE} / \mathrm{CC}(10,15,19$, $21)$, and $\mathrm{CE} / \mathrm{CE}(2,5,13,16,17,18)$.

In the experiment, match trials were considered to be those trials in which the configurations were identical. To control for possible effects of varying complexities between subsets, Match trials with the same orientation were also included to obtain baseline values. Mismatch trials always concerned the same arrangement of parts but a different configuration because the orientation of one of the parts was inverted. Thus, for each of the configurations depicted in Fig. 1C, there were six different mismatch trials (three in the depicted orientation and three in the inverted orientation). To avoid response biases, each match consisting of the same configuration, was presented six times to each participant as well (again, three in the depicted orientation and three in the inverted orientation). Therefore, the total number of stimuli to class $\times$ configurations $\times($ match + mismatch $)=3 \times 24 \times 12=864$.

\subsection{Apparatus}

The experiment was carried out on an IBM-compatible computer with a PENTIUM 133 processor. The stimuli were displayed on a $64 \mathrm{~K}$ color monitor with a refresh rate of $70 \mathrm{~Hz}$ and a $1024 \times 768$ pixel display area.

\subsection{Procedure}

Each trial started with a blank screen for $1500 \mathrm{~ms}$. After that, two configurations 
appeared on the screen, one on the left and one on the right. The task of the participants was to respond whether these configurations were identical (either in the same orientation or in inverted orientations) by pressing one of two keyboard buttons. The participants were seated approximately $1.20 \mathrm{~m}$ from the monitor screen. Each configuration subtended a visual angle of less than 2 degrees. The participants were trained with a set of 30 stimuli (i.e. a random sample from the experimental trials).

\section{Results}

We have first analyzed the data in a two-factorial class (3) $\times$ orientation (2) design with the response times (RT) of the correct matches (which were $96 \%$ of all matches) as dependent variable. An ANOVA for repeated measurements gave the following results: a main effect of class $(F(2,16)=20.58, P<0.0001)$, a main effect of orientation $(F(1,8)=45.93, P<0.0005)$, and a significant class $\times$ orientation interaction $(F(2,16)=15.15, P<0.0005)$. In addition, we have analyzed the following partial interactions. Class (connected, disconnected) $\times$ orientation $(1,2): F(1,8)=16.18, P<0.005$; class (disconnected, control) $\times$ orientation $(1,2): F(1,8)=32.29, P<0.0005$; class (connected, control) $\times$ orientation $(1,2): F(1,8)<1$ (see Fig. 2 A for a plot of the mean values).

In the following analyses, the RT values on the $0^{\circ}$ orientations are taken as baseline values. Thus, $\mathrm{RT}^{\prime}$ values are calculated in which the RT values on the $0^{\circ}$ orientations are subtracted from the RT values on the $180^{\circ}$ orientations. As additional independent variables, we consider two relevant a posteriori variables in two separate analyses: concavity (two pairs of deep concavities versus one pair of deep concavity), and type of connection (EE/EE, EE/CE, EE/CC, CE/CE).

To analyze effects of concavity, an ANOVA for repeated measurements was run in a two-factorial class $(3) \times$ concavity $(2)$ design performed on the $\mathrm{RT}^{\prime}$ values. The results were as follows: a main effect of class $(F(2,16)=15.58, P<0.0005)$, no effect of concavity $(F(1,8)=3.04, P=0.12)$, and no class $\times$ concavity interaction $(F(2,16)=1.08, P=0.36)$ (see also Fig. 2B).

To analyze effects of type of connection, an ANOVA for repeated measurements was run in a two-factorial class (3) $\times$ type of connection (4) design. The analyses were again performed on the $\mathrm{RT}^{\prime}$ values and gave the following results: a main effect of class $(F(2,16)=11.41, P<0.001)$, a main effect of type of connection ( $F$ $(3,24)=7.50, P<0.005)$, and no class $\times$ type of connection interaction $(F$ $(6,48)=1.03, P=0.42)$ (see also Fig. 2C).

\section{Discussion and conclusions}

The strong effect of orientation is in line with the classic results on mental rotation tasks (Shepard and Metzler, 1971). The baseline value (orientation $0^{\circ}$ ) was approximately the same (about $1500 \mathrm{~ms}$ ) for each stimulus class, whereas for the inverted 

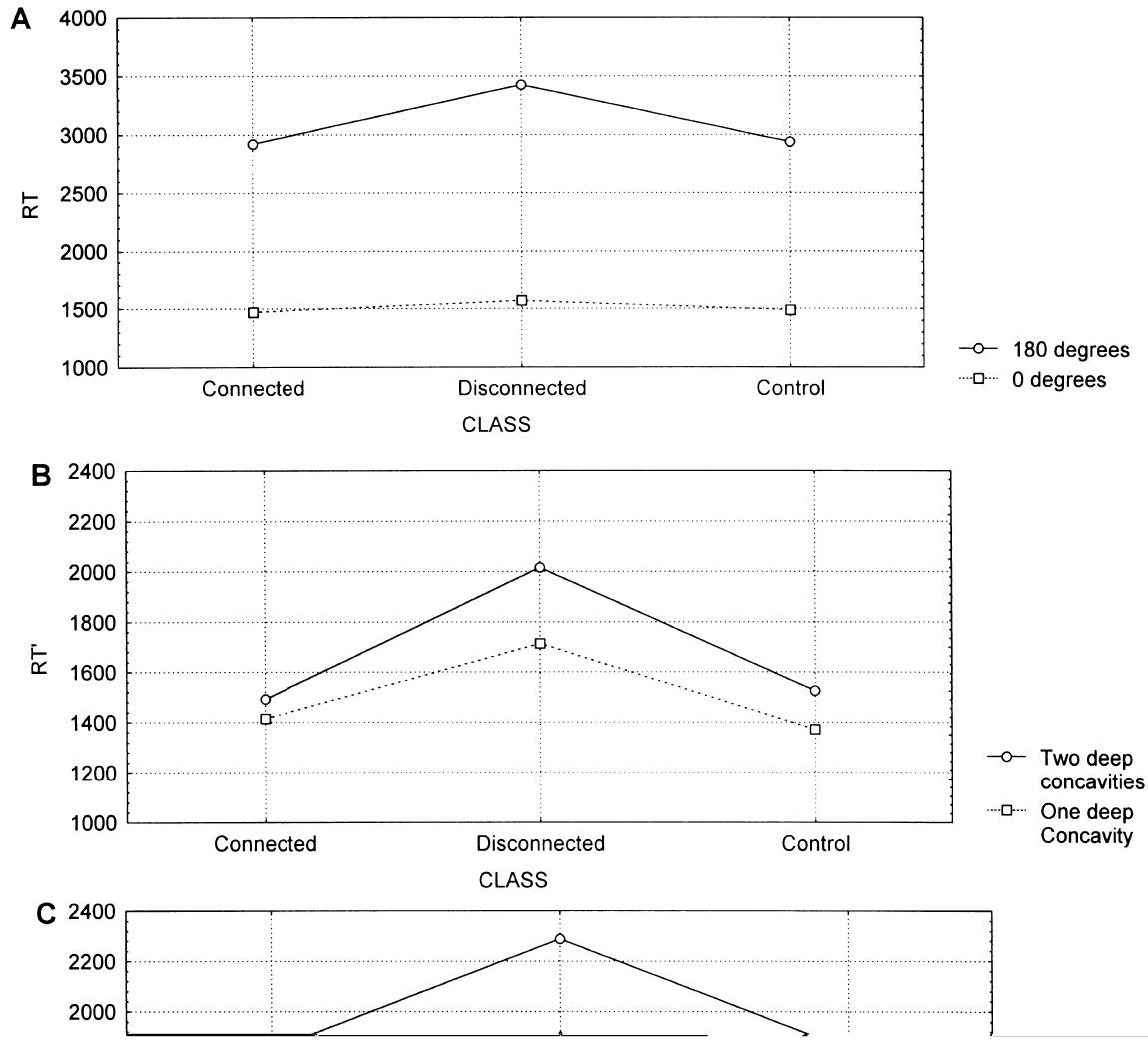

Fig. 2. (A) Mean response times for the class $\times$ orientation design. (B) Mean corrected response times (RT $180^{\circ}-\mathrm{RT} 0^{\circ}$ ) for the class $\times$ concavity design. (C) Mean corrected response times for the class $\times$ type of connection design.

orientation (i.e. $180^{\circ}$ rotated versions) the comparison times appeared to be much slower for the disconnected configurations, both in comparison with the connected and the control configurations. The results of the partial interaction analysis clearly confirmed this special status of the disconnected configurations. It is somewhat surprising perhaps that the RT values on the control configurations were almost the same as those on the connected configurations. Apparently, the higher saliency of the parts (e.g. Hoffman and Singh, 1997) or the higher complexity of the whole configuration induced by the black outlines, had no effect on the comparison times. Additionally, the second analysis showed that the effect of connectivity on the RT' 
values also appeared for those stimuli in which the parts all reveal deep concavities. On the basis of these results, it can be concluded that physical connectivity is a crucial factor in a task which requires a mental operation, like rotation or inversion, on the stimulus. We reason that the matching of inverted disconnected configurations requires more processing time as the parts are compared much more independently from each other than for the connected and the control configurations. In other words, considering the overall data, the representational unity is lowest for the disconnected configurations.

Inspecting the results of the third analysis, however, at first glance reveals a paradoxal finding. Compare, for example, the RT' values on CE/CE with those on EE/EE (Figs. 2C, see also Fig. 3 in which a connected and disconnected stimulus is shown for each of these configurations). In the $\mathrm{CE} / \mathrm{CE}$ configurations, the parts are minimally connected and are clearly distinguishable, whereas in the EE/EE configurations the edges are always completely connected. No doubt, the latter connection is much stronger (e.g., Van Lier et al., 1994). One might therefore expect that a distortion of this connection would greatly influence the RT' values. However, there was virtually no effect of (dis)connection for the EE/EE configurations; analyzing the specific contrasts showed that the difference between connected and disconnected was far from significant for the EE/EE configurations $(F(1,8)<1)$, whereas it was significant for the $\mathrm{CE} / \mathrm{CE}$ configurations $(F(1,8)=5.42, P<0.05)$.

The answer to this seeming incongruity lies in the fact that the disconnected EE/ EE configurations remain relatively integrated because of other figural properties that act as 'perceptual glue' such as the parallelism of edges between the parts. This finding does not mean that physical connection is not always effective. What it shows, in our view, is simply that its effect can be measured best when other perceptual grouping factors are minimized (which was the case in our stimulus construction). The fact that we found a reliable effect of physical connectivity for configurations with minimal physical connection (deep concavities, corner to edge connections) precisely demonstrates its representational impact. Moreover, these results strengthen the arguments made recently (Saiki and Hummel, 1998) that

\section{Connected Disconnected}

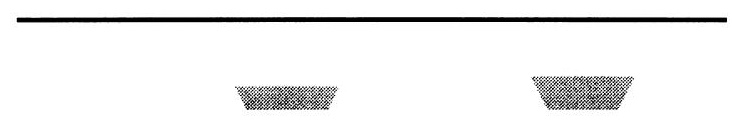

Fig. 3. A connected and a disconnected exemplar is shown for the CE/CE and EE/EE configurations. 
part based theories of object recognition, which describe objects as a collection of parts segmented at concavities (as in the RBC model, Biederman, 1987) should also include an explicit representation of the connections between the parts. Only then would one have an adequate structural description theory of object representation within which factors affecting the actual representational unity of objects could be incorporated.

\section{Acknowledgements}

This research was conducted at the Laboratory of Experimental Psychology at the University of Leuven, where R.V.L. held a Postdoctoral Fellowship from the Research Council at the University of Leuven. The final version of this paper was prepared at the Nijmegen Institute for Cognition and Information (NICI, University of Nijmegen) while R.V.L. received a personal postdoc grant ('Persoonlijke Postdoc Subsidie') from the Foundation for Social and Behavioral Sciences ('Stichting voor Maatschappij en Gedragswetenschappen') of the Netherlands Organization for Scientific Research ('NWO'). Both funding organizations are greatly acknowledged. Financial support was also given by the Research Program of the Fund for Scientific Research (Flanders, Belgium) ('FWO') to J.W. (G.0130.98). The first author is currently at the Nijmegen Institute for Cognition and Information, University of Nijmegen, The Netherlands.

\section{References}

Baylis, G., Driver, J., 1993. Visual attention and objects: evidence for hierarchical coding of location. Journal of Experimental Psychology: Human Perception and Performance 19, 451-470.

Baylis, G., Driver, J., 1995a. One-sided edge assignment in vision. 1. Figure-ground segmentation and attention to objects. Current Directions in Psychological Science 4, 140-146.

Baylis, G., Driver, J., 1995b. Obligatory edge assignment in vision: the role of figure and part segmentation in symmetry detection. Journal of Experimental Psychology: Human Perception and Performance $21,1323-1342$.

Bethell-Fox, C., Shepard, R., 1988. Mental rotation: effects of complexity and familiarity. Journal of Experimental Psychology: Human Perception and Performance 14, 12-23.

Biederman, I., 1987. Recognition by components: a theory of human image understanding. Psychological Review 94, 115-147.

Hoffman, D., Richards, W., 1984. Parts of recognition. Cognition 18, 65-96.

Hoffman, D., Singh, M., 1997. Salience of visual parts. Cognition 63, 29-78.

Hummel, J., Biederman, I., 1992. Dynamic binding in a neural network for shape recognition. Psychological Review 99, 480-517.

Kubovy, M., Wagemans, J., 1995. Grouping by proximity and multistability in dot lattices: A quantitative Gestalt theory. Psychological Science 6, 225-234.

Kubovy, M., Holcombe, A.O., Wagemans, J., 1998. On the lawfulness of grouping by proximity. Cognitive Psychology 35, 71-98.

Lamote, C., Wagemans, J., 1999. Rapid integration of contour fragments: from simple filling-in to partsbased shape description. Visual Cognition 6 (2/3) (in press).

Marr, D., Nishihara, H., 1978. Representation and recognition of three-dimensional shapes. Proceedings of the Royal Academy of London Series B 200, 269-294. 
Palmer, S., Rock, I., 1994. Rethinking perceptual organization: the role of uniform connectedness. Psychonomic Bulletin and Review 1, 29-55.

Saiki, J., Hummel, J., 1998. Connectedness and the integration of parts with relations in shape perception. Journal of Experimental Psychology: Human Perception and Performance 24, 227-251.

Siddiqi, K., Tresness, K., Kimia, B., 1996. Parts of visual form: psychophysical aspects. Perception 25, 399-424.

Shepard, R., Metzler, J., 1971. Mental rotation of three-dimensional objects. Science 171, 701-703.

Van Lier, R., Van der Helm, P., Leeuwenberg, E., 1994. Integrating global and local aspects of visual occlusion. Perception 23, 883-903.

Wertheimer, M., 1923. Untersuchungen zur Lehre von der Gestalt (Investigations for the use of the study of Gestalt). Psychologische Forschung 4, 301-350. 